\title{
Familiar words facilitate segmentation of novel similar sounding words in 7- month-olds
}

\author{
Melanie S. Schreiner ${ }^{1,2}$, Nicole Altvater-Mackensen ${ }^{3}$, and Nivedita Mani ${ }^{1,2}$ \\ ${ }^{1}$ Psychology of Language Research Group, University of Göttingen, Germany \\ ${ }^{2}$ Leibniz ScienceCampus "Primate Cognition”, Göttingen, Germany \\ ${ }^{3}$ Institute of Psychology, University of Mainz, Germany
}

\begin{abstract}
Author Note
Correspondence regarding this article should be addressed to Melanie S. Schreiner, Georg-EliasMüller Institute for Psychology, Psychology of Language Research Group, University of Göttingen, Goßlerstr. 14, 37073 Göttingen, Germany.

E-mail: melanie.schreiner@psych.uni-goettingen.de
\end{abstract}




\section{Familiar words facilitate segmentation of novel similar sounding words in 7- month-olds}

Familiarity with a word-form has been identified as one source of information infants use in detecting and segmenting individual words from continuous speech. Here we extend this finding to examine whether prior familiarity with the phonological form and meaning of a word (based on natural language exposure) influences segmentation of words from fluent speech. The current study tested infants' segmentation of pseudo-words that sound similar to words infants were likely to already be familiar with, given their natural exposure to language at home. A wordcomprehension task confirmed that infants were not only familiar with the chosen word-forms but also their meaning. Results of the segmentation task suggest that infants are able to use their knowledge of previously familiar words to segment similar-sounding words from the speech stream. Thus, word-form similarity based segmentation is a powerful mechanism that can drive infants' rapid vocabulary growth. In addition, the study confirms that 7-month-old infants already comprehend simple frequent words and are able to distinguish them from subtle mispronunciations of these words.

Keywords: word segmentation, word-form familiarity, phonological similarity, language development

word count: 6800 


\section{Introduction}

Infants as early as 7.5 months of age, if not earlier (Altvater-Mackensen \& Mani, 2013; Bortfeld, Morgan, Golinkoff, \& Rathbun, 2005; Jusczyk \& Aslin, 1995) can segment words from fluent speech streams and store these word-form representations in long-term memory such that they are able to recognize them later (Jusczyk \& Hohne, 1997; Schreiner, Altvater-Mackensen, \& Mani, 2016). These are important steps on the way to early lexical acquisition, allowing infants to develop a so-called proto-lexicon of word-forms (Swingley, 2005a) initially devoid of rich meaning representations.

The pioneering Jusczyk and Aslin (1995) study examined infants' segmentation of words from fluent speech, showing that infants listened longer to subsequently presented isolated tokens of words only when they had been presented with these words in fluent speech earlier. Notably, the authors examined infants' segmentation of potentially familiar words like cup and $\mathrm{dog}$. They controlled for this by presenting infants with other similarly familiar words as control words (that infants had no exposure to in fluent speech) and found increased listening to the familiarized than the control words. While the authors also report that infants' familiarity with the words included prior to the study was minimal (as reported by parental questionnaires), the possibility remains that infants may have heard some (or all) of the words beforehand and that this prior familiarity may have helped infants segment the words from fluent speech.

Indeed, considerable research suggests that infants' prior familiarity with words or wordforms can influence their segmentation of words from fluent speech. Thus, as early as 4 months, infants are able to detect their own name when presented with these repeatedly in lists (Mandel, Jusczyk, \& Pisoni, 1995). Prior familiarity with words also helps infants segment neighboring words from the speech stream: From around 6 months of age, highly familiar words like Mommy 
can be used as a cue to a word boundary, helping infants better segment words following such familiar words in fluent speech (Bortfeld et al., 2005). The presence of a neighboring familiar word can also help infants segment difficult words, such as iambs, from fluent speech (Sandoval \& Gómez, 2016). However, this effect of word-form familiarity on segmentation is contingent on the correctness of the pronunciation of this familiar word, with infants being unable to use the transitional probabilities of syllable sequences to segment speech, when a mispronounced familiar word is introduced into the speech stream (Mersad \& Nazzi, 2012).

Recent work extends this to examine the influence of prior familiarity with words on infants' segmentation of similar-sounding words from fluent speech. This work finds that novel words that are phonologically similar to previously familiarized word-forms are readily segmented from fluent speech by infants between 6 to 8 months of age (Altvater-Mackensen \& Mani, 2013). Here, infants were familiarized with a word, e.g., Löffel, and then tested on their segmentation of a similar-sounding word, i.e., Lökkel, from the speech stream. Infants showed improved segmentation of words that sounded similar to previously familiarized words. These experiments highlight a powerful bootstrapping mechanism available to young infants to update their lexical knowledge, such that each new word the baby learns can act as the pedestal to her learning other words that overlap in form with this new word.

However, the similar-sounding words in Altvater-Mackensen and Mani (2013) were presented immediately after infants were familiarized with the initial words. Thus, as soon as infants were familiarized with Löffel, they were presented with sentences containing the similarsounding word, Lökkel. While the study demonstrates an effect of word-form familiarity on word segmentation, the real-world impact of such bootstrapping is questionable since it is unlikely that infants are given such sequential exposure to similar-sounding words in their natural input. In other 
words, it remains unclear whether the effect of word-form familiarity on word segmentation also holds for words that are already familiar to the infant and which they have not been familiarized with directly preceding the segmentation task.

Support in favor of such a possibility comes from research suggesting that infants do not need immediate prior exposure to word-forms in isolation in order to segment these from speech. Infants appear to encode and later retrieve words they had been exposed to in their natural environment over a controlled, extended period of time (Jusczyk \& Hohne, 1997), even when this extended exposure is in the more challenging adult-directed speech register (Schreiner et al., 2016). Thus, infants appear to be able to store word-forms in long-term memory and recognize these in fluent speech later on. Indeed, this ability to encode word-forms in long-term memory presumably underlies the previously reported findings of the influence of familiar words on infant segmentation (e.g., Bortfeld et al., 2005; Mersad \& Nazzi, 2012; Sandoval \& Gómez, 2016). This leaves open the possibility that infants can use their prior familiarity with words, i.e., words they are exposed to at home, to help them segment similar-sounding words from fluent speech.

Indeed, a number of recent studies suggest that even very young infants already known the meanings of words for body parts (Tincoff \& Jusczyk, 2012) and common nouns for food and drinks (Bergelson \& Swingley, 2012, 2015, 2018). Thus, infants need not only use their prior familiarity with the form of a word to segment similar-sounding words from fluent speech, but may also rely on familiar words, whose meanings they are familiar with, in segmenting similarsounding words from fluent speech. Indeed, words whose meanings infants may be familiar with may particularly boost segmentation of similar-sounding words since it is likely that infants have more robust representations of words whose meaning and sound they are familiar with. 
Taken together, the work reviewed thus far suggests that infants are able to use 1) their prior familiarity with word-forms to segment neighboring words from fluent speech and 2) their recent familiarity with word-forms to segment similar-sounding words from fluent speech. Against the background of the literature suggesting infants' considerable familiarity with both the meaning and form of frequent words in their input, the current study brings together the findings reported above to examine whether infants are better able to segment words from fluent speech when these words sound similar to words they were previously familiar with. In other words, can infants use their prior familiarity with words like $b e d$, acquired in their natural home environments, to segment similar-sounding words, such as Beck, from fluent speech?

\section{Current study}

To examine this, we presented infants with a standard segmentation task (cf., Jusczyk \& Aslin, 1995) followed by a word comprehension task. In the segmentation task, they were presented with sentences containing novel words, e.g., Beck, that sound similar to words the infants may previously be familiar with, i.e., Bett, or sentences containing novel control words, e.g., Nipp, that did not sound similar to previously familiar words. In a subsequent test phase, infants were presented with isolated tokens of the different words, i.e., Beck and Nipp, to examine whether there were differences in listening times to either kind of word. Differences in listening times to words that sound similar to previously familiar words and words that do not sound similar to previously familiar words would provide evidence in support of our hypothesis that infants can use their prior familiarity with the word-forms of familiar words to segment similar-sounding words from fluent speech.

Once infants had completed the segmentation task, they took part in a preferential-listening task (Golinkoff, Hirsh-Pasek, Cauley, \& Gordon, 1987), where they were presented with images 
depicting prototypical referents of the familiar words (bed, diaper) alongside familiar distractor items (e.g., baby bottle), and heard the familiar label for these images (i.e., Bett, Windel). This was to assess their familiarity with the meanings of the word-forms presented. Increased looking at the target image following the auditory familiar label would suggest that infants were familiar with the referents of the presented labels.

We note that infants may listen longer to the similar-sounding words at test in the segmentation task not because they were able to segment these words from speech but rather because they were unable to discriminate these words from the already familiar words, that is they listened longer to beck because they could not discriminate beck from bed. To examine this in further detail, we also presented infants with trials where they saw the images of the referents of the familiar words (alongside the same distractors as above) and heard the similar-sounding words, i.e., beck. This is similar to mispronunciation trials in tasks examining children's sensitivity to mispronunciations of familiar words (Bergelson \& Swingley, 2012; Mani \& Plunkett, 2007; Swingley \& Aslin, 2000). Increased target fixations in familiar label relative to similar-sounding trials would indicate that children could discriminate between the familiar and similar-sounding labels.

We note here two aspects of our design that require further explanation. First, we always presented infants with the segmentation task first followed by the comprehension task. Since we were interested in tapping into how infants' long-term prior familiarity with word-forms influences their segmentation of similar-sounding words from speech, it was critical that infants were not exposed to the familiar word-forms before the segmentation task. In other words, we thereby ensured that infants did not have any short-term exposure to the familiar words before hearing the similar-sounding words in the segmentation task. Second, we note that we would ideally be able 
to test physically the same stimuli counterbalanced across infants in the similar-sounding and nonsimilar words condition in the segmentation task. Such counterbalancing is common in standard segmentation tasks (and was employed in Altvater-Mackensen \& Mani, 2013). However, for such counterbalancing to work in the context of the current task, we would need to find infants who know the word bed and do not know the word diaper yet, and compare these to a different group of infants who know the word diaper, but not the word bed yet. Given how difficult it is for parents and empirical approaches to reliably estimate individual word knowledge in such young infants, such a control is difficult, if not impossible to obtain. As a result, the current task cannot be fully counterbalanced and any conclusions need to be tempered by this constraint.

Based on previous research findings (Altvater-Mackensen \& Mani, 2013), we expect that infants may show improved segmentation of words that sound similar to already familiar words. Such a finding would critically extend the real-world applicability of word-form bootstrapping to infants' segmentation of words from fluent speech, suggesting that infants can use their prior familiarity with words to segment similar-sounding words from speech. Furthermore, if infants store word-form information with sufficient phonological detail, they should also be able to detect subtle mispronunciations of these words and hence, look longer to the labeled object when presented with the correct pronunciation of this word compared to the similar-sounding mispronunciation of this word. Of further interest is the extent to which infants' familiarity with the meanings of the familiar words influences their segmentation of similar-sounding words from speech. We hypothesize, since the representations of words whose meanings infants are familiar with may be more robust and potentially more detailed, that those infants who are more familiar with the meanings of the familiar words may be better able to segment similar-sounding words from fluent speech. 


\section{Materials and methods}

\section{Participants}

The final sample of the study comprised 28 monolingual German infants at the age of 7 months $(M=210$ days, $S D=16.56 ; 12$ girls; range: 5 months 17 days - 7 months 29 days). An additional 10 children had to be excluded from the study due to fussiness $(n=5)$, failure to complete the segmentation task $(n=2)$, maternal interference $(n=2)$, and bilingualism $(n=1)$. Infants received a t-shirt or book in return for their participation.

\section{Material and Design}

Two pseudo-words (e.g., Beck [bck], Wimse [vimsa]) that resembled words already familiar to the infant ${ }^{1}$ (i.e., Bett [bet], bed; Windel [vIndal], diaper), hereafter referred to as related words, and, two pseudo-words that did not overlap phonologically with any words known to the infant (i.e., Kaster [kaste]; Nipp [nIp]), hereafter referred to as unrelated words, were selected for the study. Four passages with six different grammatically and syntactically correct sentences were recorded by a female speaker in an infant-directed manner (see Figure S1 and Table S1 in the Appendix). Each sentence in a passage contained the same pseudo-word, so that one of the four pseudowords was repeated six times within one passage. Furthermore, the same female speaker recorded a number of isolated tokens of all four pseudo-words. Three different isolated tokens of each pseudo-word were selected to be presented to infants in the test phase. In addition, the female speaker recorded the two familiar words, Bett (bed) and Windel (diaper), as well as the two related words, Beck and Wimse, embedded in different carrier phrases to be used during the word

\footnotetext{
${ }^{1}$ Using the childes-db for North-American English (MacWhinney, 2000; Sanchez, et al., 2019, version 2018.1), we confirmed that these words occurred in interactions with 6-month-olds.
} 
comprehension task. An animated checkerboard and images of a bed, a diaper, an apple and a baby bottle were used as visual stimuli.

\section{Procedure}

\section{Word segmentation task}

Infants were seated on their parent's lap or in a car seat for the duration of the study. A monitor located $100 \mathrm{~cm}$ in front of them displayed the visual stimulus, i.e., the blinking checkerboard. Two loudspeakers located above this monitor presented the auditory stimuli of the study.

Trials in the familiarization and test phase only began when the infants were fixating the monitor in front of them. Upon fixation, an experimenter blind to condition initiated the trials, beginning with the blinking checkerboard on-screen and the presentation of the auditory stimulus. The experimenter indicated via key-press throughout the experiment whether infants were orienting towards the monitor or away. The auditory stimulus continued so long as the infant continued to fixate the monitor. Trials ended when the infant looked away from the monitor for more than $2 \mathrm{~s}$ or when the auditory stimulus was completed.

The auditory stimulus presented differed across the familiarization and the test phase of the experiment. During the familiarization phase, in each trial, infants listened to passages of sentences containing one of the four pseudowords. Each passage was 19s long. Infants were presented with passages containing all four pseudowords, i.e., words that sounded similar to previously familiar words, henceforth known as related words (Beck and Wimse) and words that do not sound similar to previously familiar words, henceforth known as unrelated words (Kaster and Nipp), during the familiarization phase across trials. The familiarization phase concluded when infants accumulated 
a total of 100 s of listening time to the familiarization passages or when all trials had been presented to infants ( $n=12,3$ passages or trials per pseudoword).

During the test phase, infants were presented with three test blocks containing four trials each with a total of 15 repetitions of isolated tokens of one of the pseudo-words in each trial. Trials continued until the end, that is $20 \mathrm{~s}$, or until infants looked away from the screen for more than $2 \mathrm{~s}$ (cf., Jusczyk \& Aslin, 1995). In each block, infants were presented with one trial each for each of the pseudo-words, i.e., two related trials and two unrelated trials, summing up to 6 related and 6 unrelated trials over the course of the test phase.

Coding and Analyses. Infants' looking behavior was assessed online using the digital stimulus presentation and scoring system Present (Meints \& Woodford, 2008) by an experimenter who observed infants' fixations of the monitor via two cameras located above the monitor. The experimenter coded whether infants were fixating the monitor by pressing a corresponding button on the keyboard. Both parents and experimenter were blinded to the experimental phase and condition (by wearing headphones) to prevent any systematic influence on infants' listening behavior. Later, the coding output was aligned with information about the phase and the condition of the experiment. A second independent coder also assessed 14\% of the analyzed data to confirm the reliability of the coded data with a high degree of inter-rater reliability, $r=0.95$.

For each infant, we calculated the mean listening times during the test phase to trials presenting the isolated tokens of the related and unrelated words. A difference in infants' listening times aggregated separately for related and unrelated trials would be taken as an index of infants' discrimination of these words.

We report two kinds of analyses. First, we compared listening times during related and unrelated trials using a paired samples t-test. Second, we also examined performance in the 
segmentation task using linear mixed effects models with the lmer4 function in R. This allows us to better capture the natural variance in different infants' responding to different target items across the course of the experiment, in comparison to standard t-tests which do not consider these critical aspects of potential variance in the data (Baayen, Davidson, \& Bates, 2008). A first model compared listening times in the test phase across the related and unrelated word conditions. We included condition (related, unrelated word) as a fixed effect and Participant id and the nested effect of Participant id: item as random effects. To evaluate the effect of condition on infants' listening times, this full model was compared to a reduced model which did not include condition. Finally, in order to compare performance across the different related words, we ran an additional model using only those trials where the related words were presented comparing listening times across the two kinds of related words, i.e., with item as a fixed effect.

\section{Word comprehension task}

We tested infants' comprehension of the meanings of the two familiar words by presenting them with images depicting one of the two words, i.e., bed/diaper, side-by-side on screen with a familiar distractor item, i.e., apple/baby bottle for the duration of 5000ms. We chose to pair bed and diaper with alternative distractor images rather than with one another because infants could solve the task where they were paired together with knowledge of just one word (and not the other) by exclusion-based reasoning ("I know that's a bed, so the other one must be a diaper").

Using a carrier phrase, infants were directed to look at the familiar object with the onset of the target label appearing at $2000 \mathrm{~ms}$, followed by a single instance of the target label in isolation at 4000ms. Each familiar word was tested twice with target side being counterbalanced across trials. The order of familiar words tested was counterbalanced across children. The image of the familiar word bed was always paired with the familiar distractor item apple, whereas the image of 
the diaper was always paired with the baby bottle. Finally, to ensure that infants were able to discriminate the related word, e.g. Beck, from the familiar word, e.g. Bett, we also presented infants with trials where they saw the two familiar images and heard the related pseudo-words, i.e., Beck and Wimse. Within the word comprehension task, infants were first presented with the familiar words followed by the similar-sounding pseudo-words.

Coding and Analyses. A Tobii eye-tracker automatically provided data for infants' fixations every $8 \mathrm{~ms}$ frame of a trial. Based on this, we separately calculated the proportion of time that infants spent looking at the target object before it was named, hereafter pre-naming phase, and after it was named, hereafter post-naming phase, by dividing the time infants spent looking at the target by the time infants spent looking at both, target and distractor. Thus, for each test trial, we separately calculated the proportion of target looking (hereafter, PTL) for the pre- and post-naming phase. The pre-naming window counted all eye-movements that took place one second before the onset of the target word, whereas the post-naming window included all eye-movements beginning with a standard delay of $240 \mathrm{~ms}$ after the onset of the target word to account for the time required to respond to a stimulus (e.g., Swingley \& Aslin, 2000) till the end of the trial at 5000ms. An increase in fixations to the target object from the pre- to the post-naming phase is taken as an index of word recognition. If infants are able to discriminate the pseudo-words from the familiar words, there should be a difference in the increase in PTL from the pre- to the post-naming phase between correct pronunciation trials and mispronunciation trials (cf., Swingley \& Aslin, 2002 for the general design of a mispronunciation task). This helps control whether a preference for the related words in the segmentation task could be due to infants not being able to discriminate the related words from the familiar words. 
Again, we report two kinds of analyses. A first traditional analysis examines the increase in PTL from the pre-naming to the post-naming phase in the correct and mispronunciation trials using a paired samples t-test. The dependent variable in these analyses is the baseline corrected PTL (where the PTL in the pre-naming window is subtracted from the PTL in the post-naming window on a trial-by-trial basis) separated by condition (correct and mispronunciations).

Next, we used growth curve models (for a more detailed description and instruction see Mirman, 2016; Mirman, Dixon, \& Magnuson, 2008) to examine the change in participants' fixations to the target across the time course of the trials. Growth curve models have recently proved advantageous in comparing non-linear change in infants' looking behavior across the entire time-course of a trial (see Eiteljoerge, Adam, Elsner, \& Mani, 2019 for a theoretical discussion motivating the reported GCA). In contrast to radically reduced mean fixation proportions across the entire time window, growth curve analysis allows us to model differences across the time course.

The dependent variable in these analyses is the baseline-corrected PTL in each time-point in the post-naming window (from 2240 to $5000 \mathrm{~ms}$ ), where the PTL aggregated across the baseline window is subtracted from the PTL in each time-point in the post-naming window on a trial-bytrial basis (see Eiteljoerge et al., 2019 for the same approach). We routinely include a baseline correction in all our analyses since infants may have a perceptual visual preference for either of the objects presented on-screen which typically manifests in the baseline phase and we only want to consider changes in target fixations relative to this baseline phase in our final analyses to ensure that we only consider eye-movements that can reasonably be construed a response to the auditory target, rather than a continued manifestation of an early visual preference. 
We fitted a generalized mixed model using lme4's lmer function in R (Bates, Mächler, Bolker, \& Walker, 2015) with Gaussian error structure and identity link function. A first model included Time and its second and third polynomial allowing us to capture linear, quadratic and cubic curvatures across the time-course. We also included linear and quadratic time terms as random slopes across participants and items. A reduced model was fit which did not include condition and was compared to a full model including the interaction between condition (correct, mispronunciations) and the time terms.

Finally, to examine the influence of word comprehension abilities on performance in the segmentation task, we added the baseline-corrected PTL (aggregated across the entire post-naming window) in the model examining performance in the segmentation task (see above) and compared this to a model excluding this factor.

\section{Results}

\section{Segmentation task}

A paired samples t-test comparing listening times in the related and the unrelated condition found that participants listened longer to unrelated trials, $M=12.06, S D=3.35$, relative to related trials, $M=10.98, S D=3.22, t(27)=2.56, p=.016, d=.48$ (see Table 1 and Figure 1). Similarly, adding condition to the model examining listening times improved the fit of the model, $\chi^{2}(1)=4.367$, $p=.037$, confirming the difference in looking times to related versus unrelated trials. A separate model examining listening times in only related trials found no significant effect of item, with no difference in listening times to the two items, $\chi^{2}(1)=.19, p=.66$. 
A similar analysis on listening times in the familiarization phase found no difference in listening times to related and unrelated trials, $t(27)=-.15, p=.88, d=.029$. Adding condition similarly did not improve model fit, $\chi 2(1)=.0013, p=.971$, suggesting no significant differences in listening times for passages containing the related target words, $M=14.62, S D=4.36$, and passages containing the unrelated target words, $M=14.47, S D=3.83$. At the very least, any differences in the subsequent test phase cannot be reduced to different amounts of exposure to sentences containing the targets in the familiarization phase.

\section{Word comprehension task}

First, we examined whether there was a difference in baseline-corrected target fixations in the post-naming window across correct $(M=0.08, S D=0.48)$ and mispronunciation trials $(M=0.04$, $S D=0.50)$ and found no significant difference between the two trial types, $t(20)=-.86, p=.4, d=.18$. Thus, when averaging target fixations across the entire time window, we found no difference between the two trial types.

Next, we examined the growth curve model modeling target fixations across the time course of the trial and found that adding condition (correct, mispronunciation) improved the fit of the model with an effect of condition on the slope, $\chi^{2}(4)=9.96, p=.041$, with some suggestion that infants looked longer at the target when presented with correct pronunciations of the label for this image relative to incorrect pronunciations (see Table 2 and Figure 2). In the correct pronunciation condition, infants showed an increase in fixations of the target, while, in the mispronunciation condition, infants showed a minimal preference for the target object with a decrease to chance in the last $1500 \mathrm{~ms}$.

Separate models examined performance in the correct pronunciation condition and the mispronunciation condition with third-order orthogonal polynomials. Again, we added random 
effects at the level of the participants to account for variability between participants. In particular, we included linear and quadratic time terms as random slopes across participants. For the model examining the correct pronunciations, the linear and quadratic time terms were significant suggesting that infants correctly fixated the target more upon being presented with the label for this image. As shown in Figure 2, participants started off at chance increasing fixations to the target before beginning a return to chance towards the end of the trial. There was a significant effect on the cubic time term for the model examining mispronunciations, as shown by initial rise in fixations to the target followed by a drop to chance and a potential small rise towards the end of the trial. Table 3 shows the fixed effects parameter estimates and their standard errors along with the $\mathrm{p}$-values estimated using the normal approximation for $\mathrm{t}$-values.

We then calculated the increase in fixations to the target from the baseline to the postnaming window in the word-comprehension task. Adding this measure of word comprehension to the model examining the segmentation data did not improve model fit, $\chi^{2}(1)=1.06, p=.303$. In other words, we found no evidence to support the hypothesis that infants who were more familiar with the meanings of the familiar words were better able to segment similar-sounding words from fluent speech.

\section{Discussion}

The current study set out to examine whether infants were able to use their prior familiarity with words (based on their natural exposure to language at home) to segment similar-sounding words from fluent speech. Previous work reports that infants who had recently been familiarized with word-forms in the laboratory were better able to segment words sounding similar to these pre-familiarized words from fluent speech (Altvater-Mackensen \& Mani, 2013). Here, we extend this to the more naturalistic situation of examining the effect of infants' natural prior familiarity 
with words on their segmentation of words sounding similar to these familiar words from fluent speech.

The results of the word comprehension task provide modest evidence in support of the finding that infants were already familiar with the meanings of our chosen familiar words. Infants looked longer at the target picture when presented with the correct familiar label. Importantly, infants were able to discriminate the familiar words from subtle mispronunciations of these words, showing longer fixations to the target only when the words were correctly pronounced and not when presented with the related words used in the segmentation task. These findings are in line with previous work on 5-month-olds' mispronunciation detection in which infants listened longer to correct pronunciations of their own name compared to vowel mispronunciation in a preferential listening task (Bouchon, Floccia, Fux, Adda-Decker, \& Nazzi, 2015). Figure 2 suggests that infants in the current study initially fixated the target object upon hearing the target label in both the correct pronunciation and the mispronunciation condition. However, starting around 1000ms after the onset of the target word, infants in the correct pronunciation condition increased their fixations to the target object whereas infants in the mispronunciation condition initially showed an increase in target fixations followed by a decline to chance towards the rest of the trial. This observed looking pattern is in keeping with our expectations, given the overlap at the onset of the correct and mispronounced target words. The correct and mispronunciations overlap at onset and diverge upon presentation of the (word-medial) coda. Thus, it would be fair to assume that infants initially fixate the target and only later move to the distractor upon processing the mismatching (word-medial) coda, which has already been observed in older children (see Swingley, 2009; Swingley, Pinto, \& Fernald, 1999). The fact that fixations to the correct and mispronunciation diverge later in the time course is therefore most likely due to the words differing from the (word- 
medial) coda, and due to the much younger age of the children tested in the current study. In terms of the segmentation task, which we discuss next, this suggests, at the very least, that infants were able to discriminate the familiar words from the similar-sounding words in the context of referents of these words.

In addition, the results of the segmentation task suggest that prior familiarity with a word infants learned in their natural home environment helps them segment similar sounding words from fluent speech: Infants listened longer to novel unrelated words (Nipp, Kaster) relative to the related words (Beck, Wimse), i.e., the words that sounded similar to the familiar words (Bett, Windel). This presents considerable insights to the real-world applicability of word-form bootstrapping to infants' segmentation of words from speech. Altvater-Mackensen and Mani (2013) familiarized infants with words and immediately tested their segmentation of similarsounding words from fluent speech. While these findings highlight a powerful mechanism available to young infants segmenting words from speech, it is unlikely that infants are exposed to similar-sounding words in similarly quick succession in their natural environment. This raised the question of the extent to which infants can use words they have learned at home to help segment similar-sounding words from fluent speech. The current study shows that even very young infants can use the few words they know to help them segment similar sounding words from fluent speech. Infants may, therefore, use existing phonological representations in learning new word-forms (Storkel, 2001; Storkel \& Hoover, 2011). Indeed, the acquisition of new object-word mappings appears to be influenced by phonological similarity, where children learn novel object-word mappings easier if these words are phonologically similar to other words that they already know (Newman, Samuelson, \& Gupta, 2008). Here, we show that the acquisition of subsequent word- 
forms may similarly be facilitated by word-forms already established in the infants' proto-lexicon (Swingley, 2005b).

Note that infants in the current study showed a novelty preference for the novel unrelated words, while infants in Altvater-Mackensen and Mani (2013) showed a familiarity preference for words which sounded similar to the lab-familiarized words. While infants' preferences have been shown to shift from a familiarity to novelty preference in such tasks, we note that one explanation for these findings might be the difference in infants' familiarity with the familiar words critical to the two studies. Whereas the familiar words in Altvater-Mackensen and Mani (2013) were introduced for a 100 s prior to the segmentation task, infants in the current study may have had the opportunity to be confronted with the familiar words every single day starting from birth until the test date at the laboratory leading to potentially increased familiarity with the familiar words chosen in the current study. This may have driven their preference away from a familiarity preference (for words that sound similar to words they have ample experience with) to a novelty preference (for words they have not heard before, cf., Hunter \& Ames, 1988).

We note that we cannot necessarily rule out other factors that may potentially have contributed to the pattern of responding reported here. In particular, we note that previous studies on infant segmentation typically counterbalance the words presented to infants as test and control words. Thus, half the infants are exposed to one set of words in the segmentation phase and the other half of infants to a different set of words in the segmentation phase. Test and control words are then counterbalanced: words that are presented in the segmentation phase for half the infants are presented as control words for the other half of the infants and vice versa. This was not possible in the current study due to the modulation under question here, namely, that we required that infants are familiar with words that sound similar to the critical words to-be segmented. A similar 
counterbalancing in this case would require that half the infants were familiar with a set of words that the other half were unfamiliar with. Not only is this unlikely at the age tested, it is also difficult if not impossible for parents or empirical studies to accurately estimate the words known to a particular infant at this age.

Given this constraint, one possibility is that infants showed the reported pattern of responding due to a preference for the words presented, rather than due to their similarity or lack thereof to previously familiar words. In other words maybe infants just happened to like the words Nipp and Kaster better than Beck and Wimse. However, given that we find no differences in listening time during familiarization and no effect of the specific words presented to infants in the analyses reported and given that the results in previous experiments may similarly be biased by different infants having different preferences for particular sets of words, we suggest that our results are unlikely to be solely driven by such preferences. Nevertheless, we acknowledge that further research is needed to disentangle the effects of word-form familiarity on infants' word segmentation from other moderators. Given the number of words known to infants at this age, possibilities to test this are limited. One possibility available is exploring the effects of word-form familiarity on infants' word segmentation abilities in infants of other language backgrounds which may reveal further evidence for this kind of bootstrapping mechanism.

The results of the word comprehension task suggest that infants at this young age show modest familiarity with the meanings of the familiar words "bed" and "diaper" and are able to discriminate these words from subtle mispronunciations of these words. This further suggests that infants need not just have relied on simple word-form familiarity but that having a conceptual representation of these familiar words may also have contributed to the successful segmentation of the similar sounding word embedded in sentential context and its later recognition as isolated 
token. However, given that we did not find any significant correlations between infants' performance in the comprehension and segmentation task, we cannot make claims with regards to the contribution of word-meaning knowledge to word segmentation. Note again that the absence of an effect of word-meaning knowledge on segmentation does not imply that prior familiarity with a word-form does not impact segmentation. Indeed, prior familiarity with the form of a word - and not its meaning - has already been shown to impact segmentation (Altvater-Mackensen \& Mani, 2013). Here, we suggest that - within the constraints of the task discussed above - such prior familiarity also extends to words that children have heard in their natural environment.

While the results of the word comprehension task suggest that children did not confuse the related words for their real-word counterparts, it remains open if they confused these two kinds of words in the segmentation task. Thus, one possibility remains that while children did not confuse the two words in the word recognition task, given the additional boost provided by the visual images, children may have confused the words in the segmentation task. We note, however, that this was tested by Altvater-Mackensen and Mani (2013) with no evidence for the suggestion that children were merely confusing the words in the test phase of the segmentation task. Furthermore, even if infants were merely confusing mispronounced words with already familiar words, we suggest that this could be the first stages of the bootstrapping process, steering attention to the related words in ways that words without such related neighbors would not receive.

Another caveat of the current study is that the prevalence of such similar-sounding words may be rather low in the infant input, raising questions as to the psychological validity of such a finding. However, given that infants are also able to segment words from overheard speech (cf., Schreiner et al., 2016) where the incidence of such similar-sounding words may be higher, the opportunities offered by such a bootstrapping mechanism may be greater. Indeed, the prevalence 
of clusters of phonologically related words in the infant lexicon (cf., Mani \& Plunkett, 2010, 2011b) raise the possibility that such a mechanism may even contribute to the development of early word links. Furthermore, while the current study examined words that overlap considerably, it remains to be seen whether a similar mechanism operates when words overlap to a lesser extent (in cases of graded phonological similarity, e.g., Mani \& Plunkett, 2011a), thereby showcasing the additional contribution of this mechanism to word learning.

Indeed, we found no evidence for such graded phonological effects in the current study. To the extent that one can interpret a null result, we found no difference in the listening times to more closely related pairs (Beck-Bett) and more distantly related pairs (Windel-Wimse). This is surprising since if the findings reported above capture the influence of word-form familiarity on segmentation, one might have expected this influence to be modulated by the phonological similarity of the words involved. On the other hand, it remains to be seen whether infants are capable of showing such graded phonological effects at the ages tested. Furthermore, the finding that previous studies find similar effects of word-form familiarity regardless of whether the phonological overlap is at the onset or the offset of the words suggests that there may not be as much fine-grained detail involved in such bootstrapping (cf., Altvater-Mackensen \& Mani, 2013).

In sum, the current study finds modest evidence in support of the finding that infants as young as 7 months already comprehend simple words and demonstrates that they are able to distinguish them from subtle mispronunciations of these words. For the purposes of the current study, here we extend previous findings of an influence of word-form familiarity on infants' word segmentation to words infants have learned in their natural home environment. Taken together with the findings of Altvater-Mackensen and Mani (2013), the results of the current study suggest that the words a child knows can influence the words that she is likely to acquire next. In other 
words, each word the child knows can act as a stepping stone to her acquisition of other related words. 


\section{Acknowledgements}

This work was founded by the German Excellence Initiative Award to the University of X (Third funding line: Institutional Strategy). We thank parents and babies for their participation in the study.

\section{Data availability statement}

The data that support the findings of the current study are openly available on OSF at https://osf.io/6tch9/?view only=6b7e9a7c2e2549f98ce03099fb9e2f36. 


\section{References}

Altvater-Mackensen, N., \& Mani, N. (2013). Word-form familiarity bootstraps infant speech segmentation. Developmental Science, 16(6), 980-990.

Baayen, R. H., Davidson, D. J., \& Bates, D. M. (2008). Mixed-effects modeling with crossed random effects for subjects and items. Journal of Memory and Language, 59(4), 390-412.

Bates, D., Mächler, M., Bolker, B., \& Walker, S. (2015). Fitting linear mixed-effects models using lme4. Journal of Statistical Software, 67, 1-48.

Bergelson, E., \& Swingley, D. (2012). At 6-9 months, human infants know the meanings of many common nouns. Proceedings of the National Academy of Sciences, 109(9), 3253-3258.

Bergelson, E., \& Swingley, D. (2015). Early word comprehension in infants: Replication and extension. Language Learning and Development, 11(4), 369-380.

Bergelson, E., \& Swingley, D. (2018). Young infants' word comprehension given an unfamiliar talker or altered pronunciations. Child Development, 89(5), 1567-1576.

Bortfeld, H., Morgan, J. L., Golinkoff, R. M., \& Rathbun, K. (2005). Mommy and me: Familiar names help launch babies into speech-stream segmentation. Psychological Science, 16(4), 298-304.

Bouchon, C., Floccia, C., Fux, T., Adda-Decker, M., \& Nazzi, T. (2015). Call me Alix, not Elix:

Vowels are more important than consonants in own-name recognition at 5 months. Developmental Science, 18(4), 587-598.

Eiteljoerge, S. F., Adam, M., Elsner, B., \& Mani, N. (2019). Word-object and action-object association learning across early development. PLOS ONE 14(8): e0220317.

Golinkoff, R. M., Hirsh-Pasek, K., Cauley, K. M., \& Gordon, L. (1987). The eyes have it: Lexical and syntactic comprehension in a new paradigm. Journal of Child Language, 14(1), 23-45. 
Hunter, M. A., \& Ames, E. W. (1988). A multifactor model of infant preferences for novel and familiarstimuli. Advances in Infancy Research, 5, 69-95.

Jusczyk, P. W., \& Aslin, R. N. (1995). Infants' detection of the sound patterns of words in fluent speech. Cognitive Psychology, 29(1), 1-23.

Jusczyk, P. W., \& Hohne, E. A. (1997). Infants' memory for spoken words. Science, 277(5334), 1984-1986.

MacWhinney, B. (2000). The CHILDES Project: Tools for analyzing talk. Third Edition. Mahwah, NJ: Lawrence Erlbaum Associates.

Mandel, D. R., Jusczyk, P. W., \& Pisoni, D. B. (1995). Infants' recognition of the sound patterns of their own names. Psychological Science, 6(5), 314.

Mani, N., \& Plunkett, K. (2007). Phonological specificity of vowels and consonants in early lexical representations. Journal of Memory and Language, 57(2), 252-272.

Mani, N., \& Plunkett, K. (2010). In the infant's mind's ear: Evidence for implicit naming in 18month-olds. Psychological Science, 21(7), 908-913.

Mani, N., \& Plunkett, K. (2011a). Does size matter? Subsegmental cues to vowel mispronunciation detection. Journal of Child Language, 38(3), 606-627.

Mani, N., \& Plunkett, K. (2011b). Phonological priming and cohort effects in toddlers. Cognition, 121(2), 196-206.

Meints, K., \& Woodford, A. (2008). Lincoln Infant Lab Package 1.0: A new programme package for IPL, Preferential Listening, Habituation and Eyetracking. [WWW document: Computer software \& manual]. Available from http://www.lincoln.ac.uk/psychology/babylab.htm 
Mersad, K., \& Nazzi, T. (2012). When Mommy comes to the rescue of statistics: Infants combine top-down and bottom-up cues to segment speech. Language Learning and Development, $8(3), 303-315$.

Mirman, D. (2016). Growth curve analysis and visualization using R. Boca Raton, FL: CRC Press.

Mirman, D., Dixon, J. A., \& Magnuson, J. S. (2008). Statistical and computational models of the visual world paradigm: Growth curves and individual differences. Journal of Memory and Language, 59(4), 475-494.

Newman, R., Samuelson, L., \& Gupta, P. (2008). Learning novel neighbors: Distributed mappings help children and connectionist models. Proceedings of the Annual Meeting of the Cognitive Science Society, 30(30), 29-34.

Sanchez, A., Meylan, S. C., Braginsky, M., MacDonald, K. E., Yurovsky, D., \& Frank, M. C. (2019). childes-db: a flexible and reproducible interface to the Child Language Data Exchange System. Behavior Research Methods, 51(4), 1928-1941.

Sandoval, M., \& Gómez, R. L. (2016). Overriding the metrical bias with lexical information: English-learning 7.5-month-olds use Mommy to segment iambic words. Language Learning and Development, 12, 398-412.

Schreiner, M. S., Altvater-Mackensen, N., \& Mani, N. (2016). Early word segmentation in naturalistic environments: Limited effects of speech register. Infancy, 21(5), 625-647.

Storkel, H. L. (2001). Learning new words: Phonotactic probability in language development. Journal of Speech, Language, and Hearing Research, 44(6), 1321-1337.

Storkel, H. L., \& Hoover, J. R. (2011). The influence of part-word phonotactic probability/neighborhood density on word learning by preschool children varying in expressive vocabulary. Journal of Child Language, 38(3), 628-643. 
Swingley, D. (2005a). 11-month-olds' knowledge of how familiar words sound. Developmental Science, 8(5), 432-443.

Swingley, D. (2005b). Statistical clustering and the contents of the infant vocabulary. Cognitive Psychology, 50, 86-132.

Swingley, D. (2009). Onsets and codas in 1.5-year-olds' word recognition. Journal of Memory and Language, 60(2), 252-269.

Swingley, D., \& Aslin, R. N. (2000). Spoken word recognition and lexical representation in very young children. Cognition, 76(2), 147-166.

Swingley, D., \& Aslin, R. N. (2002). Lexical neighborhoods and the word-form representations of 14-month-olds. Psychological Science, 13(5), 480-484.

Swingley, D., Pinto, J. P., \& Fernald, A. (1999). Continuous processing in word recognition at 24 months. Cognition, 71(2), 73-108.

Tincoff, R., \& Jusczyk, P. W. (2012). Six-Month-Olds comprehend words that refer to parts of the body. Infancy, 17(4), 432-444. 


\section{Appendix}

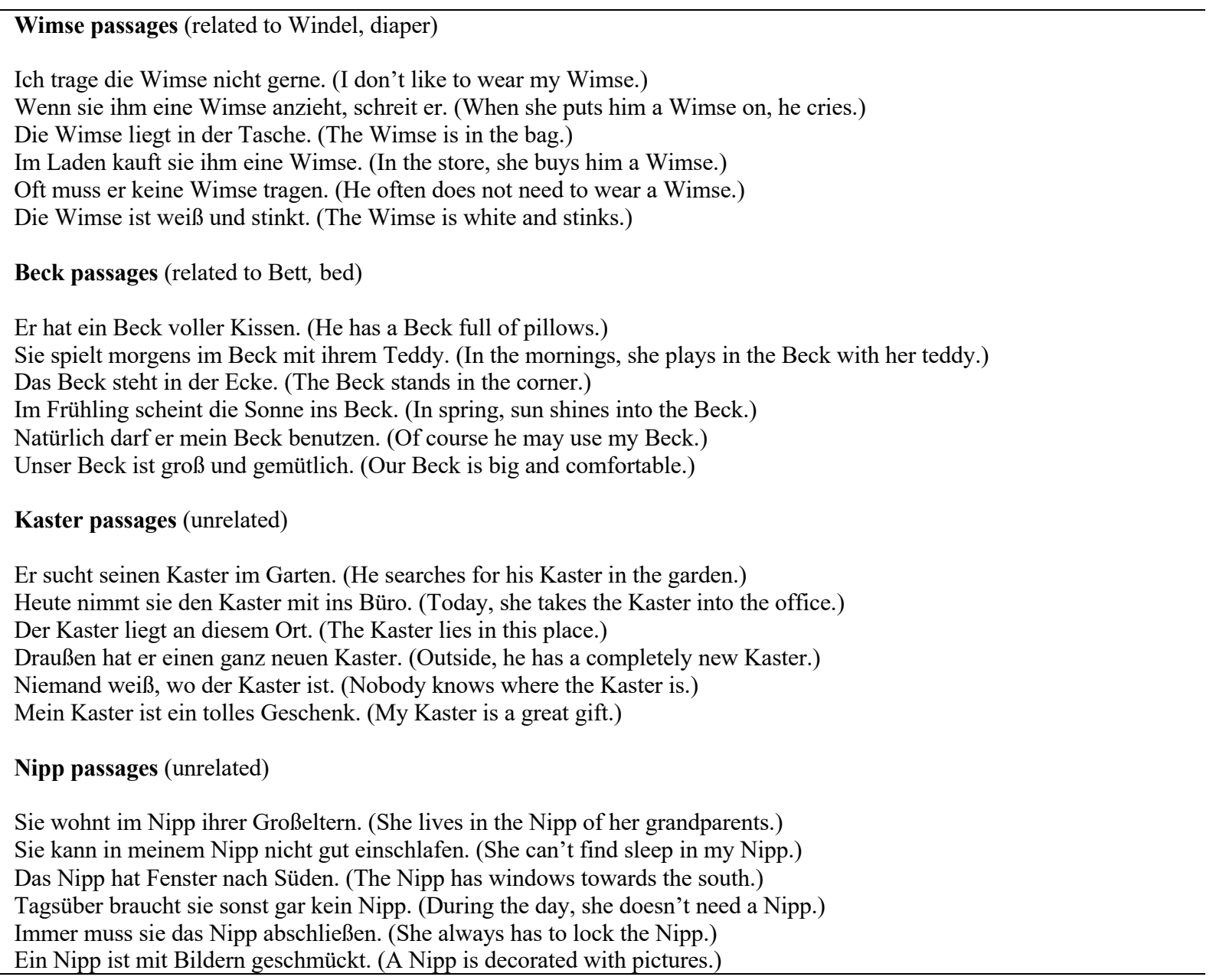

Figure S1. Auditory stimuli of the familiarization phase of the word segmentation task. 
Table S1 Mean, minimum, and maximum fundamental frequency in $\mathrm{Hz}$ and mean duration in $\mathrm{ms}$ for the passages and isolated tokens of the study. Standard deviations are provided in brackets. For the recordings of the exaggerated German IDS stimuli, a female native speaker of German imagined herself as speaking to a child. In addition, she was asked to produce the passages and isolated tokens in a slower and more exaggerated way than she typically would.

\begin{tabular}{lllll}
\hline & mean F0 & min F0 & max F0 & mean duration \\
\hline passages & $226(12)$ & $112(39)$ & $365(34)$ & $2688(339)$ \\
isolated tokens & $238(49)$ & $180(37)$ & $293(107)$ & $774(220)$ \\
\hline
\end{tabular}




\section{Tables}

Table 1. Mean listening times in seconds for each word and each phase. Standard deviations are provided in brackets.

\begin{tabular}{lcc}
\hline & Familiarization phase & Test phase \\
\hline Beck & $13.91(5.41)$ & $11.05(5.96)$ \\
Wimse & $13.65(5.71)$ & $10.68(5.50)$ \\
Nipp & $13.73(5.88)$ & $12.60(5.76)$ \\
Kaster & $13.71(6.23)$ & $11.77(5.56)$ \\
\hline
\end{tabular}


Table 2. Results of the growth curve model testing the effect of condition on infants' preferential target looking over time.

\begin{tabular}{lcccc}
\hline & Estimate & $\boldsymbol{S E}$ & $\boldsymbol{t}$-value & $\boldsymbol{p}$-value \\
\hline Intercept & -0.013 & 0.047 & -0.281 & 0.778 \\
Linear & -0.022 & 0.156 & -0.139 & 0.889 \\
Quadratic & 0.051 & 0.237 & 0.218 & 0.828 \\
Cubic & 0.132 & 0.056 & 2.348 & 0.019 \\
Condition.Correct & 0.096 & 0.045 & 2.101 & $0.035^{*}$ \\
Linear: Condition.Correct & 0.265 & 0.129 & 2.05 & $0.041^{*}$ \\
Quadratic: Condition.Correct & -0.215 & 0.317 & -0.679 & 0.497 \\
Cubic: Condition.Correct & -0.151 & 0.076 & 1.98 & $0.048^{*}$ \\
\hline
\end{tabular}


Table 3. Results of the growth curve model testing the effect target word on infants' preferential target looking over time for the correct and mispronunciation condition.

\begin{tabular}{lcccccccc}
\hline & \multicolumn{3}{c}{ Correct pronunciation } & \multicolumn{3}{c}{ Mispronunciation } \\
& Estimate & $S E$ & $t$-value & $p$-value & Estimate & SE & $t$-value & $p$-value \\
\hline Intercept & 0.081 & 0.044 & 1.84 & 0.065 & 0.040 & 0.057 & 0.706 & 0.480 \\
Linear & 0.267 & 0.136 & 1.96 & $0.049^{*}$ & -0.167 & 0.171 & -0.977 & 0.328 \\
Quadratic & -0.235 & 0.110 & -2.141 & $0.032^{*}$ & 0.053 & 0.132 & 0.397 & 0.691 \\
Cubic & -0.030 & 0.049 & -0.611 & 0.541 & 0.153 & 0.053 & 2.892 & $0.003^{*}$ \\
\hline
\end{tabular}


Figures

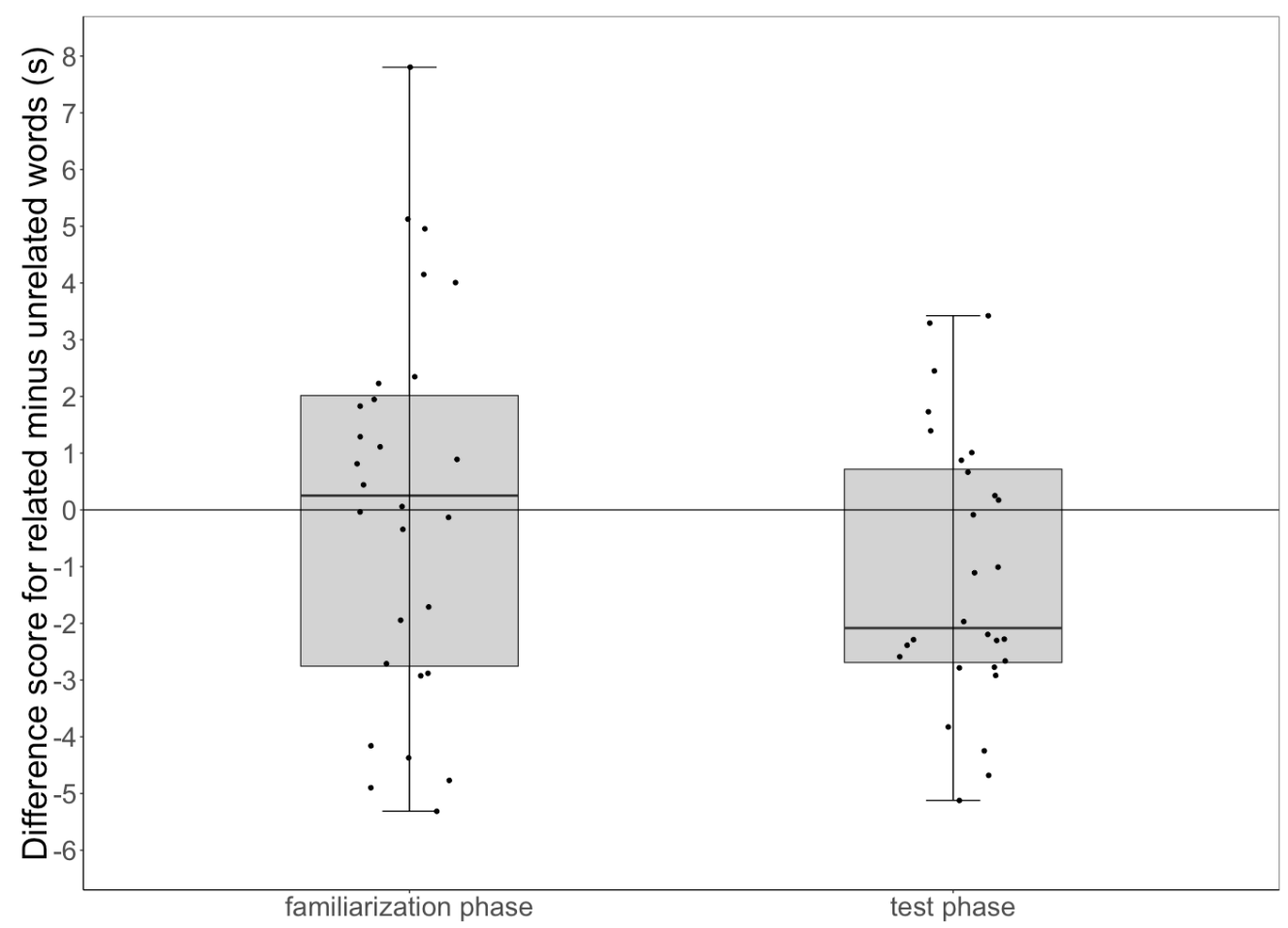

Figure 1. Difference scores for infants' listening times in seconds for related minus unrelated words during the familiarization and test phase. Dots represent individual data points. 


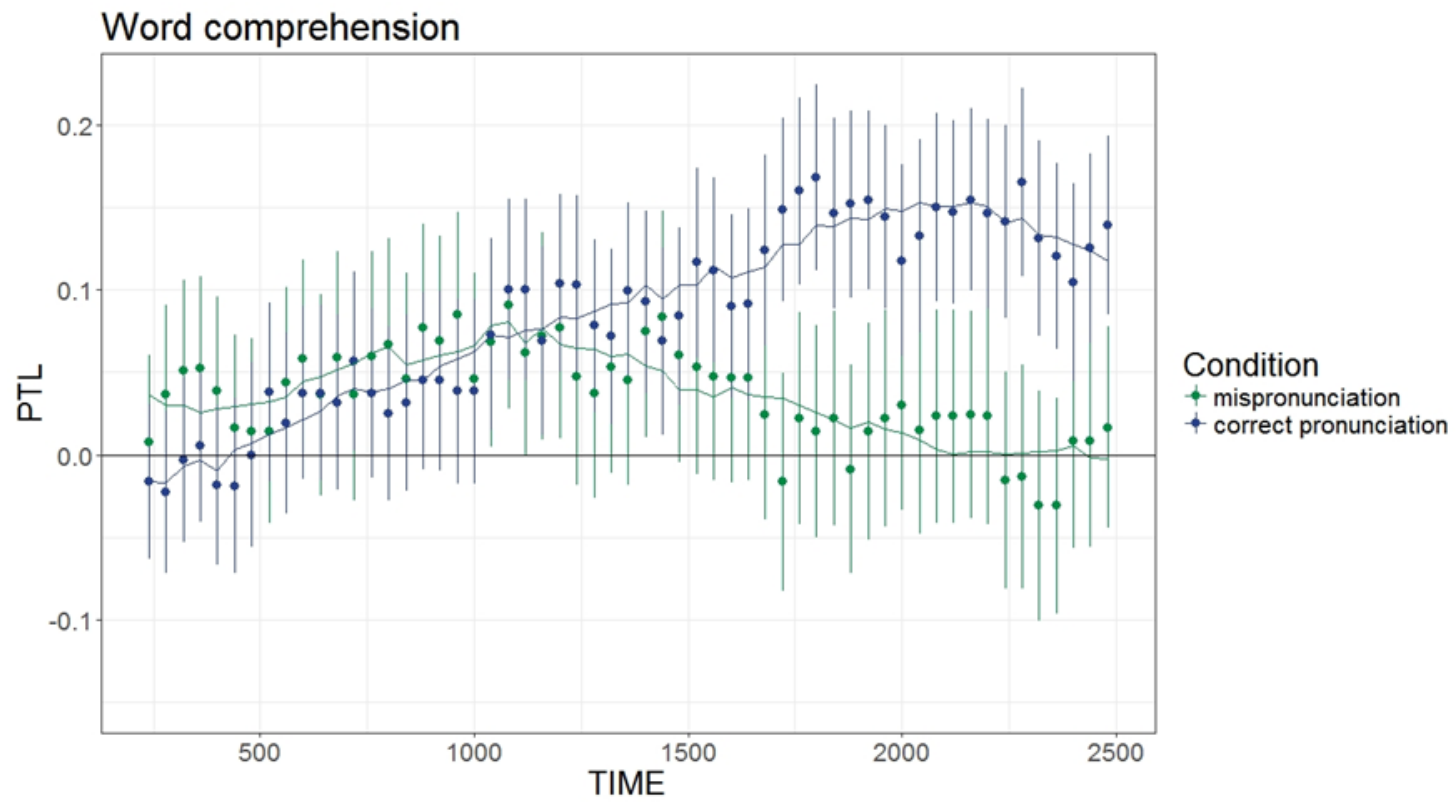

Figure 2. Time course graphs for the correct pronunciation and mispronunciation condition plotting participants' baseline-corrected PTL after word onset at 2000ms and fixation time of $240 \mathrm{~ms}$. The correct pronunciation condition is plotted in black, the mispronunciation condition in blue. The line at 0 represents chance level, everything above the line indicates target looking whereas everything below indicates distractor looking. The blue and black lines represent the fitted GLMM. 